\title{
RELIABLE AND REDUNDANT RTK POSITIONING FOR APPLICATIONS IN HARD OBSERVATIONAL CONDITIONS
}

\author{
M. Bakuła, R. Pelc-Mieczkowska, M. Walawski \\ University of Warmia and Mazury, Poland \\ e-mails: mbakula@uwm.edu.pl, renata.pelcmieczkowska@gmail.com,walawski.mariusz@olsztyn.eu
}

\begin{abstract}
It is well known that RTK (Real Time Kinematic) positioning is a very efficient technique for determination of coordinates in real time, directly on location. Although this technique has been well known since the mid-nineties of the last century, the common use of this technique developed since permanent reference GNSS (Global Navigation Satellite Systems) stations started operating as the national reference systems. Positioning in real time is very convenient for users who do not need to know any advanced technique of post-processing, especially in cases when no obstructions exist around the measured point exist. However, in practice, there are some situations when the use of RTK technique makes some difficulties, especially if the GNSS receiver has no full availability of satellites. Obstructions caused by trees, buildings, power lines etc. limit satellite availability and in consequence decrease the reliability of determined coordinates significantly. In those situations gross errors of even meters can appear in RTK positioning. In order to avoid misleading coordinates occurring we can use more than one RTK receiver simultaneously. The paper presents an approach to the RTK technology based on the simultaneous use of three different RTK receivers. Three different GNSS/RTK receivers can be set on a special mounting beam and additionally RTK positions are sent in real time to a computer. The computer software analyses not only the precision but also checks the accuracy and reliability of the RTK positions determined. Consequently, the new approach to RTK survey presented can allow obtaining reliable coordinates of centimeter accuracy even under very severe forest conditions.
\end{abstract}

Keywords: GNSS, VRS, RTK, RTCM, NMEA

\section{INTRODUCTION}

Nowadays permanent reference stations are common in many countries. They can reduce distant-dependent errors in the carrier phase and pseudorange measurements at the user station significantly (Dai et. al., 2001). But the accuracy can decrease if inter-station distances are longer than recommended (Wang et al., 2010). Many studies have indicated that accuracy of RTK positioning is at the level of better than $3-\mathrm{cm}$ in horizontal position and $1-5 \mathrm{~cm}$ in height (e.g. Hu et al., 2003; Giménez et al., 2011), when no obstructions exist around the user's observation site. Furthermore, the accuracy is significantly lower under the forest canopy and under obstructed sky (Hasegawa and Yoshimura, 2003; Lee and Ge, 2006; Bakuła et al., 2009; Pirti et al., 2010). Avoiding terrain obstructions on the survey point that have negative influence on solving the ambiguity of phase measurements represents the fundamental condition for obtaining accurate coordinates. Presence of terrain obstructions, in 
case of excessively short measurement sessions, even in case of fixed baselines solutions, may result in the situation where gross errors of even meters may appear (Bakuła et al., 2011). The degradation of accuracy under forest conditions is caused by lowering the signal strength and multipath effects while electromagnetic wave is penetrating the branches and canopies of trees. Some experiments conducted under forest conditions indicated that fixed ambiguity resolution does not guarantee high accuracy of GPS/GLONASS measurements under canopy. What is more, very high precision characterizes the results of such measurements and that is why there is no possibility of finding out a priori whether gross error occurred or not in any particular situation (Bakuła et al., 2009). Traditional RTK positioning methods provide no independent control because there is only one vector determined when using single station resolution as well as when using a virtual reference station (Vollath et al., 2002). We can say that errors in GPS measurements are the function of full environment in which the measurements are acquired (Firuzabardi and King, 2011). Nowadays the main challenge of RTK positioning is not only how to obtain precise positions but how to make obtained positions reliable, especially in obstructed environments. The questions stated by Verhagen (2004) and by Teunissen and Verhagen (2008) in their paper concerning integer ambiguity fixing and validation, are the most important nowadays, especially in hard observational conditions. In open space RTK positioning is efficient rapid technique for control points' coordinates determination (Bakuła et al., 2011). Gross errors in coordinates can be removed e.g. by the use of network differential code positioning (Bakuła, 2010).

\section{RTK TESTS PERFORMED WITH THE USE OF THREE GNSS RECEIVERS AT THE SAME TIME FOR A ONE CONTROL POINT}

The test surveys were conducted using three GNSS (Topcon Hiper Pro) receivers mounted on the specially designed linear base set up on the tripod. The design of the base allows setting three GNSS receivers on one tripod in such a way that one of them is leveled and centered directly above the surveyed point while the other two are at the distance of exactly $0.5 \mathrm{~m}$ from it and all the receivers are at the same height and form a straight line (Fig. 1). Additionally, thanks to the installed compass, the receivers are positioned in the north - south line (as a consequence the marking of the receiver applied further herein: N-North, MMiddle, S-South). The use of such a base allows introduction of the additional, independent control based on three assumptions: fixed distance of receivers from one another, their setting in line and their identical height position. Analyzing GNSS receivers on the market, we can find that JAVAD company offers the unique JAVAD TRIUMPH-4X RTK receiver, based on four GNSS antennas (www.javad.com). However, the solution presented in this paper use three GNSS receivers, which can be manufactured by different company. Additionally, the presented approach is also convenient for reliable ultra rapid static surveys (Bakuła, 2012). Although three GNSS receivers are used in this approach, we need only one GPRS module for receiving RTCM data from a reference base station. During the test surveys, RTK data from a VRS station (Virtual Reference Station) were received via the GPRS terminal. Next, the results of measurements generated at $1 \mathrm{~s}$ interval were transmitted in the NMEA standard to the computer via serial cables (Fig. 1). All the information of importance from the perspective of the further analyses is contained in the NMEA standard GPGGA message that contains, among others, the determined coordinates, the information on the GPS solution and the DOP coefficient value (Fig. 2). All the positions, independent of the solution type, were recorded in the computer. Connections between NORTH, MIDDLE, SOUTH receivers and the computer are also possible with the use of Bluetooth technology. In that case a mobile computer can be in a distance of about ten meters from the RTK receivers. 
Field measurements were carried out for two days (8 and 15 April 2009). Every day, six hour measuring session was conducted. Coordinates of the measured point, located in the middle of the forest, were known previously based on classical surveying methods. The position of survey was characterized by a high density of obstructions, caused by trees (this time without leaves) (Fig. 3). Therefore the measuring conditions during GPS observations were very difficult.

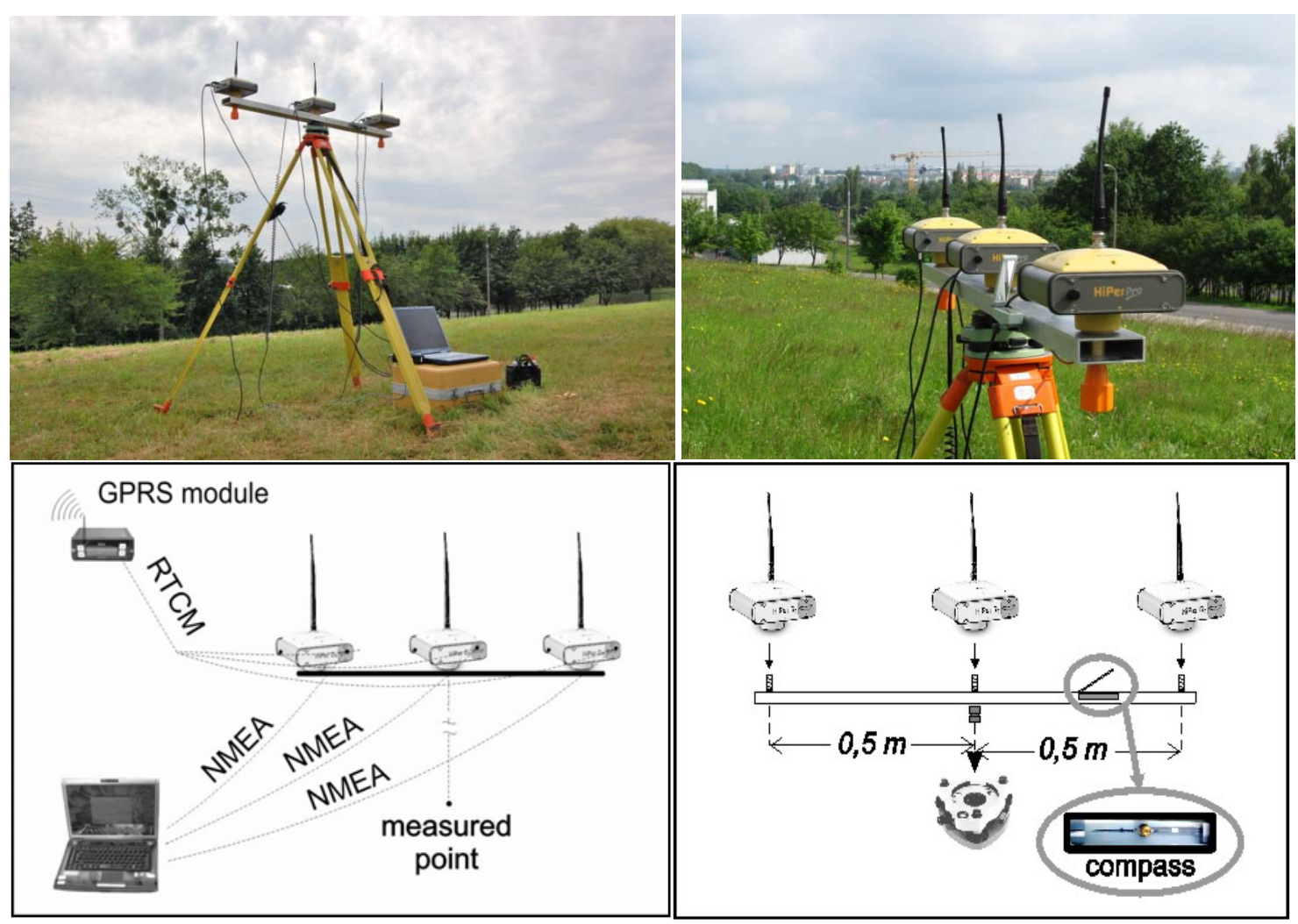

Fig. 1. Data transmission diagram and GPS/GLONASS receivers taking part in field tests

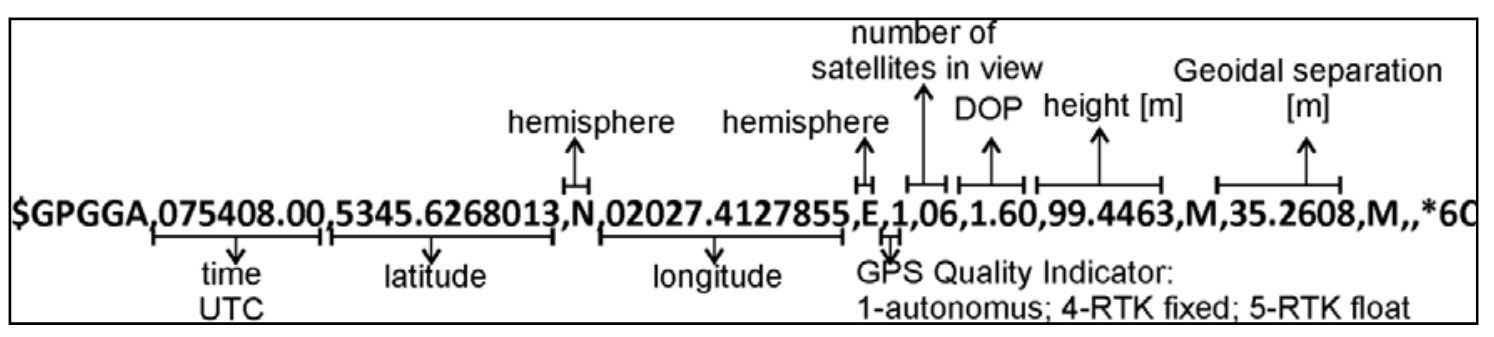

Fig. 2. GGA format of NMEA message 

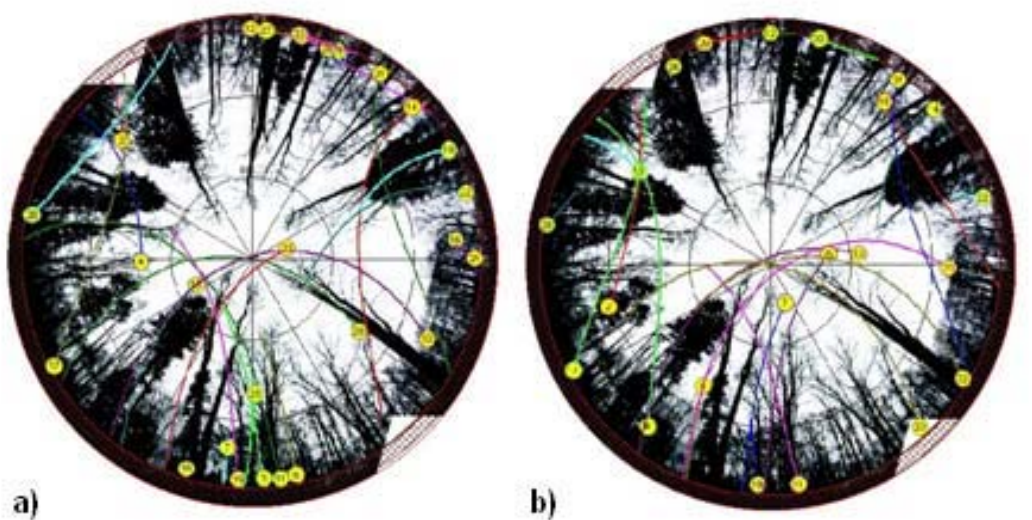

Fig. 3. Satellite trajectories and obstructions over the measured point during the RTK tests: a) April 8, 2009; b) April 15, 2009

During the experiments, there over 137 thousand positions were recorded, but only 6,297 positions were obtained with integer ambiguities. The positions with fixed solutions were arranged into separate groups with a scatter value of about a few centimeters for every group (Fig. 4). On the first day of measurements there were 23, 29 and 26 groups with fixed solution for the receivers North, Middle and South, respectively. Similarly, on the second day there were 24, 14 and 23 groups. Distributions of horizontal and vertical coordinates of points in each group, are shown in Figures 4 and 5. On each day of measurement, there were 15 and 8 groups for the Middle receiver, where the precision of coordinates in groups differed from no more than $5 \mathrm{~cm}$ horizontally and to $10 \mathrm{~cm}$ vertically. However, the accuracy of the groups was a few meters horizontally (Fig. 4) and up to several meters in height (Fig. 5).

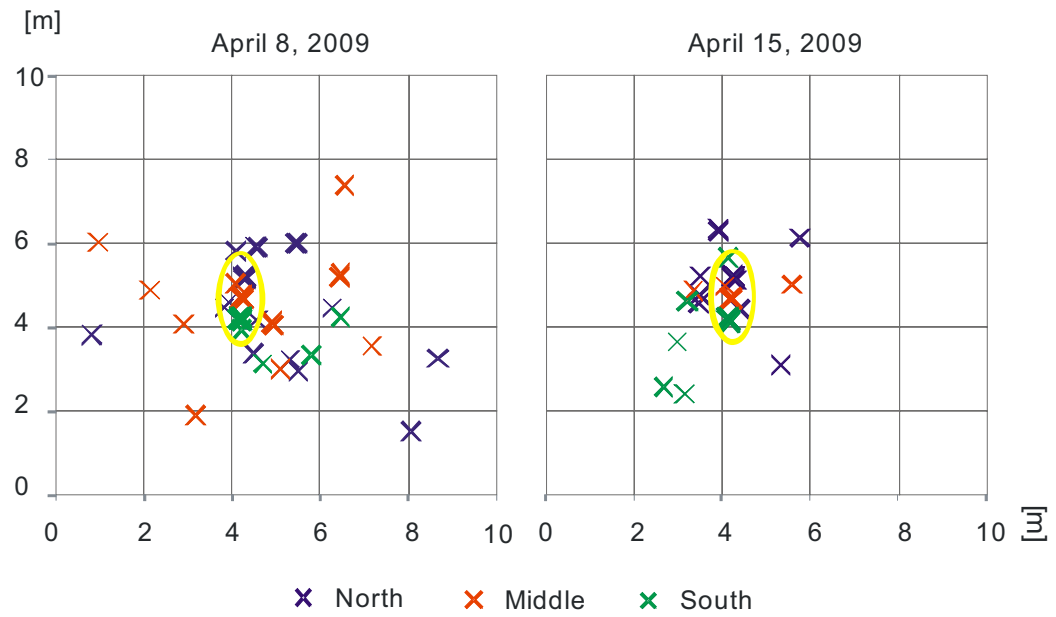

Fig. 4. Horizontal distribution of RTK positions obtained from North, Middle and South GPS/RTK receivers 


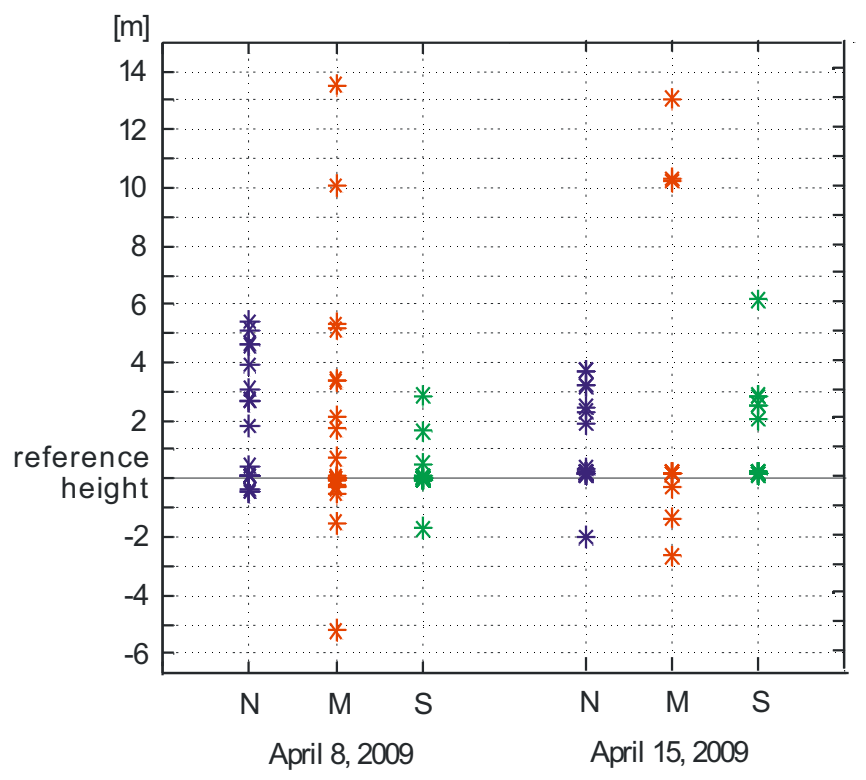

Fig. 5. Vertical distribution of RTK positions

In such difficult conditions of observation we cannot see any correlation between RTK solutions, even though the receivers were working at the same time. So we can say that in RTK positioning in the forest, the accuracy estimated by the GPS receivers present only a temporary precision. To obtain reliable RTK position repeatability of coordinates after reinitialization of RTK receivers is required. Another way of reliable solution may be to use three different GPS receivers placed at the fixed distance between each other. From a practical point of view, time is important to obtain accurate and reliable solution. The use of multiple measurements based on the assumption that RTK coordinates can be regarded as reliable when obtained after three times re-initialization as recommended in RTK positioning for difficult conditions, allows detecting gross errors (Bakuła et al., 2009) but also increases the measurement time significantly. On the other hand, use of three receivers mounted on the designed base allows reliable control of RTK survey within a shorter time (Tab. 1).

Table 1. Time for obtaining reliable coordinates using one or three GPS/RTK receivers simultaneously

\begin{tabular}{|c|c|c|c|c|c|c|}
\hline \multirow[t]{2}{*}{ Date } & \multicolumn{3}{|c|}{$\begin{array}{l}\text { Triple survey using one GPS/RTK } \\
\text { receiver }\end{array}$} & \multicolumn{3}{|c|}{$\begin{array}{l}\text { Survey conducted using three } \\
\text { GPS/RTK receivers }\end{array}$} \\
\hline & $\mathrm{dl}[\mathrm{m}]$ & $\mathrm{dh}[\mathrm{m}]$ & time hh:mm:ss & $\mathrm{dl}[\mathrm{m}]$ & $\mathrm{dh}[\mathrm{m}]$ & time hh:mm:ss \\
\hline April 4, 2009 & 0,009 & 0,018 & $00: 51: 41$ & 0,006 & 0,037 & $00: 36: 53$ \\
\hline April 15, 2009 & 0,029 & 0,073 & $04: 02: 49$ & 0,023 & 0,091 & $02: 26: 59$ \\
\hline
\end{tabular}

On the base of Table 1 we can see that application of three receivers for determination of one point coordinates shortens significantly the time of survey for reliable RTK surveys under difficult observation conditions. During those experiments no correlation between receivers in determination of the fixed position was observed; the numbers of fixed type positions determined also differed (Fig. 6) appearing at different times. The percentage values presented in Figure 6 represent the number of fixed type solutions. 


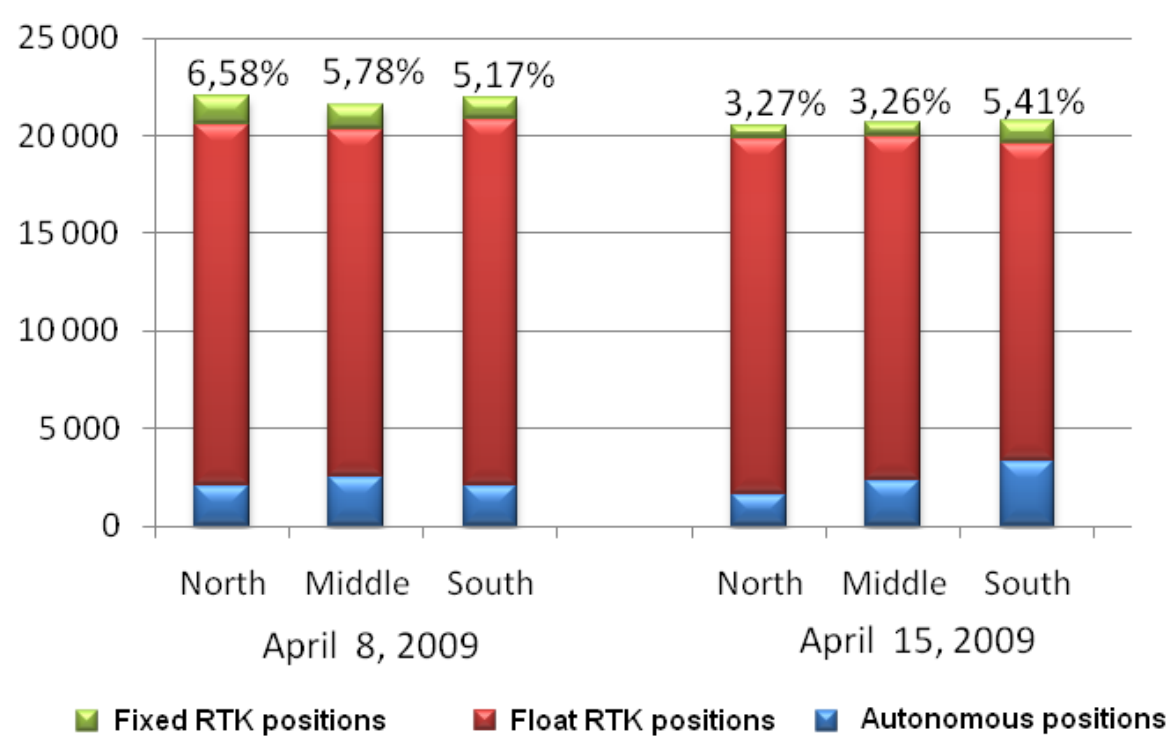

Fig. 6. Structure of the survey results obtained

On the base of Fig. 6 we can see that during the entire survey session only a few percent of the coordinates possessed the fixed solution. It is not so simple then to obtain the correct solution under the forest conditions. That is why with three GPS receivers available we have the possibility of determining reliable coordinates using combinations of solutions based on two RTK receivers if one of the three receivers had not generated the correct solution.

\section{RTK-Net APPROACH}

Considering the tests conducted using new RTK receivers it was noticed that those receivers cope with determination of the fixed type position even under very difficult observation conditions although their reliability is low. After the detailed analysis of RTK positions under forest conditions, the next step of the studies involved development of the algorithms and building the applications based on them for the purpose of reliable RTK positioning. As a result the RTK-Net application was developed that allows receiving the position from three RTK receivers and verifies the reliability of the coordinates determined. As a consequence, in the proposed model of determining the position by means of the RTK method under forest conditions three absolutely different RTK receivers are used. Three GNSS receivers set on a special beam are subject to conditions that must be met to obtain reliable coordinates of the central receiver:

- consistency of height,

- consistency of distance,

- consistency of north-south orientation.

The application developed allows connection of any GNSS receiver that sends the positions in the NMEA format to the serial port. Although we observe three GNSS receiver, as the effect we obtain just one position, i.e. coordinates of the point above which the receivers were centered, that is the coordinates of the central receiver. As a consequence of the fact that we have three receivers, we obtain redundant observations. Not always, however, satisfying all the conditions is possible. It may happen that one of the receivers is much slower with determining the fixed position. That is why in the application tested the possibility of determining the reliable position using only two of the three receivers was included. The possible combinations of the true and redundant solutions are presented in Figure 7. In case of applying just two receivers the reliable solution can be obtained in case of various 
configurations of the GNSS receivers, e.g.: NORTH and SOUTH (Fig. 7b), MIDDLENORTH (Fig. 7c) or MIDDLE-SOUTH (Fig. 7d).

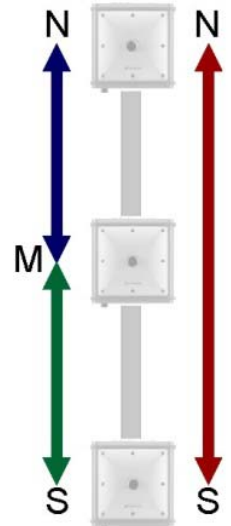

a)

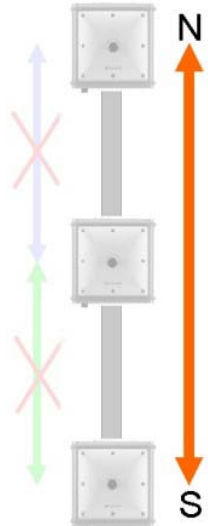

b)

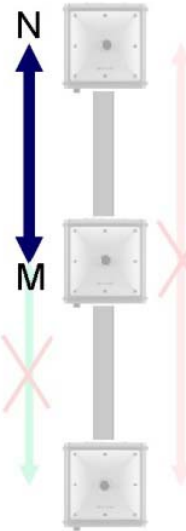

c)

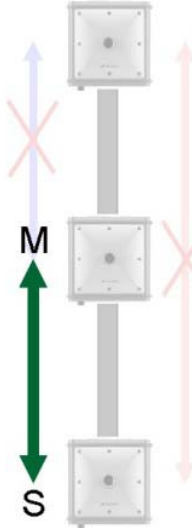

d)

Fig. 7. Diagram of the possible variants of obtaining the solution in the RTK-Net application

The test surveys of the solution developed were conducted under diversified forest conditions with different obstructions. Exemplary surveys were conducted on the $16^{\text {th }}$ and $28^{\text {th }}$ of April 2011 on the same point (Fig. 8) on which the test experiments had been conducted earlier. On the first survey date the trees surrounding the points were still without foliage while on the second survey date buds of developing leaves were present on the trees, which increased the intensity of obstructions over the point. Both survey sessions lasted for four hours and were conducted during the best possible constellation of the GPS satellites. During the survey the VRS data from the ASG-EUPOS system (Bosy et al. 2007) on the base of the GPS system satellites were used. The RTK positions determined by the GPS receivers were recorded immediately by a laptop type computer and in real time analyzed by the RTK-Net application, which informed the user about obtaining the reliable position determination. The survey experiment was conducted in such a way that each time after obtaining the information on determining the reliable coordinates the receivers were switched of and the application was reset so that each determination can be considered independent. During both survey sessions the total of over 21,000 positions were recorded of which 838 coordinates were those for which the ambiguity was solved that is the fixed type positions represented $4 \%$ of the results only. 

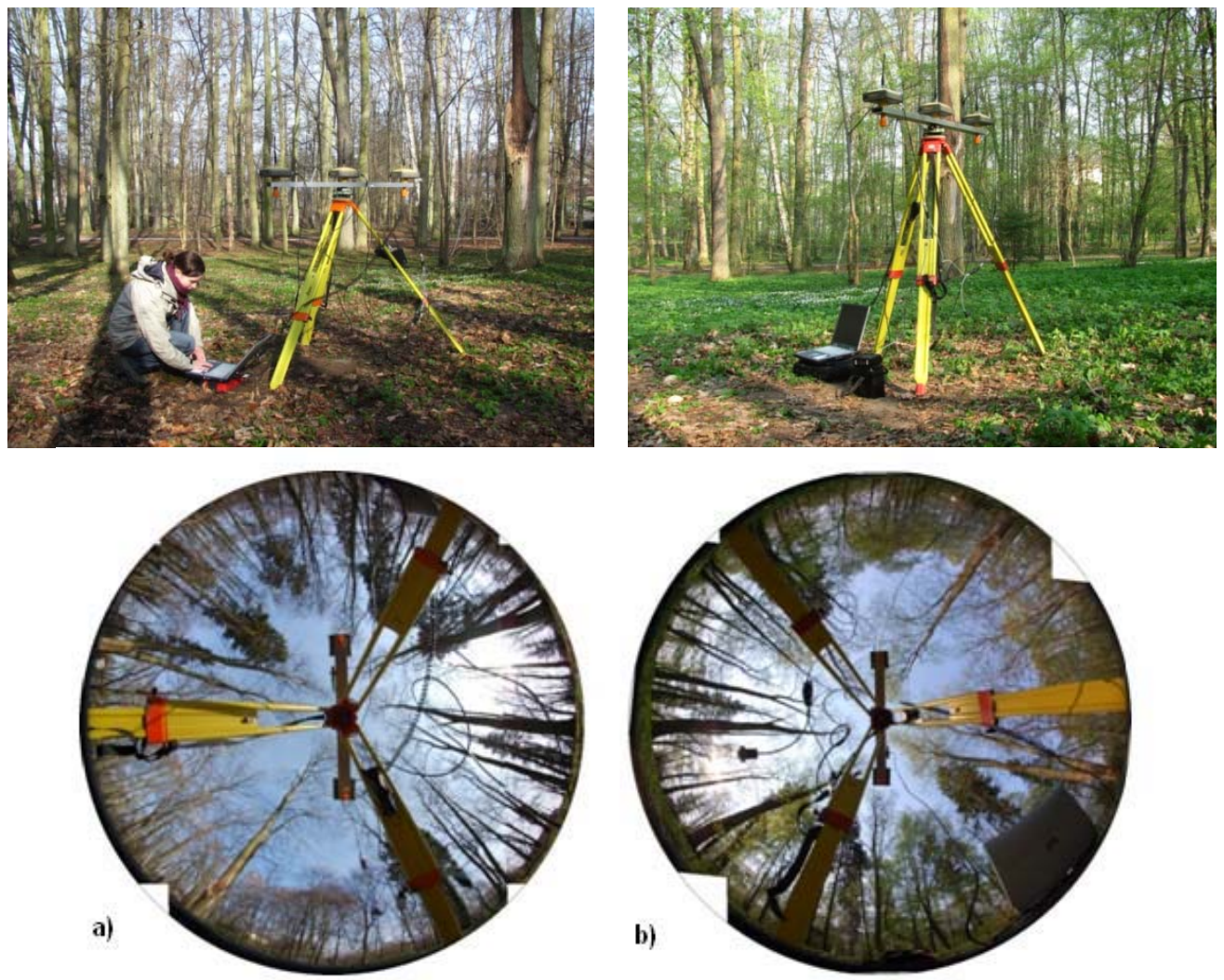

Fig. 8. Obstructions above the survey point a) April 16, 2011; b) April 28, 2011

Thanks to the application of the RTK-Net software, during every survey day reliable coordinates the accuracy of point position in case of which did not exceed $4 \mathrm{~cm}$ horizontally and $5 \mathrm{~cm}$ vertically in case of such difficult observation conditions were obtained four times independently using solution with three receivers (Tab. 2). The waiting time for such determination was from 4 to 48 minutes.

Table 2. Statistics of individual determinations of reliable coordinates

\begin{tabular}{|c|c|c|c|c|c|c|c|c|c|c|}
\hline \multirow{3}{*}{ Data } & \multirow{3}{*}{$\begin{array}{l}\text { No of } \\
\text { session }\end{array}$} & \multirow{2}{*}{\multicolumn{3}{|c|}{$\begin{array}{l}\text { Number of } \\
\text { „fixed” groups }\end{array}$}} & \multicolumn{3}{|c|}{3 receivers } & \multicolumn{3}{|c|}{2 receivers } \\
\hline & & & & & \multirow{2}{*}{$\begin{array}{l}\text { T3*) } \\
{[\mathrm{min}]}\end{array}$} & \multirow{2}{*}{$\begin{array}{c}\mathrm{dl} \\
{[\mathrm{m}]}\end{array}$} & \multirow{2}{*}{$\begin{array}{l}\mathrm{dh} \\
{[\mathrm{m}]}\end{array}$} & \multirow{2}{*}{$\begin{array}{l}\left.\mathrm{T}^{* *}\right) \\
{[\mathrm{min}]}\end{array}$} & \multirow{2}{*}{$\begin{array}{c}\mathrm{dl} \\
{[\mathrm{m}]}\end{array}$} & \multirow{2}{*}{$\begin{array}{l}\mathrm{dh} \\
{[\mathrm{m}]}\end{array}$} \\
\hline & & $\mathrm{N}$ & $\mathrm{M}$ & $\mathrm{S}$ & & & & & & \\
\hline \multirow{4}{*}{ 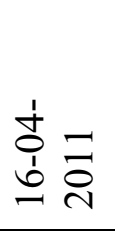 } & 1 & 1 & 27 & 13 & 32 & 0,031 & 0,036 & 20 & 0,064 & 0,097 \\
\hline & 2 & 1 & 41 & 2 & 37 & 0,032 & 0,054 & 32 & 0,052 & 0,024 \\
\hline & 3 & 1 & 5 & 14 & 4 & 0,010 & 0,045 & 1,5 & 0,032 & 0,039 \\
\hline & 4 & 8 & 16 & 1 & 40 & 0,010 & 0,021 & 1,5 & 0,032 & 0,003 \\
\hline \multirow{4}{*}{ 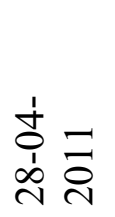 } & 1 & 2 & 7 & 9 & 30 & 0,010 & 0,044 & 15 & 0,032 & 0,030 \\
\hline & 2 & 20 & 10 & 1 & 13 & 0,032 & 0,063 & 5 & 0,032 & 0,042 \\
\hline & 3 & 15 & 1 & 13 & 28 & 0,015 & 0,018 & 17 & 0,035 & 0,021 \\
\hline & 4 & 10 & 1 & 2 & 58 & 0,015 & 0,017 & 38 & 0,035 & 0,013 \\
\hline
\end{tabular}

${ }^{*}$ T3 - time until obtaining a reliable solution based on the RTK-Net application and three GPS/RTK receivers; ${ }^{* *} \mathrm{~T} 2$ - time until obtaining a reliable solution based on two GPS receivers.

Additionally, reliable coordinates were determined using only two GNSS receivers (Tab. 2); in those cases the time until reliable determination of coordinates for the point surveyed was from $1,5 \mathrm{~min}$ up to 35 minutes. In case of using two receivers only the accuracy of the 
coordinates determined deteriorated to ca. $5 \mathrm{~cm}$ horizontally and up to $10 \mathrm{~cm}$ vertically. Although obtaining the position using three receivers provided the most reliable solution, the solutions obtained from two independent RTK receivers were already reliable in case of satisfying the conditions concerning the height, distance and mutual orientation of the RTK receivers. Use of three receivers for that technique is recommended as in that case we have one redundant receiver available. The major task of the developed application was to inform the user about obtaining the coordinates considered reliable. For the purpose of survey results reliability evaluation at the level of the application the above described conditions, i.e. the linear distance between points determined by the individual receivers equal to $0.5 \mathrm{~m}$, their positioning on the common line and equal height were verified.
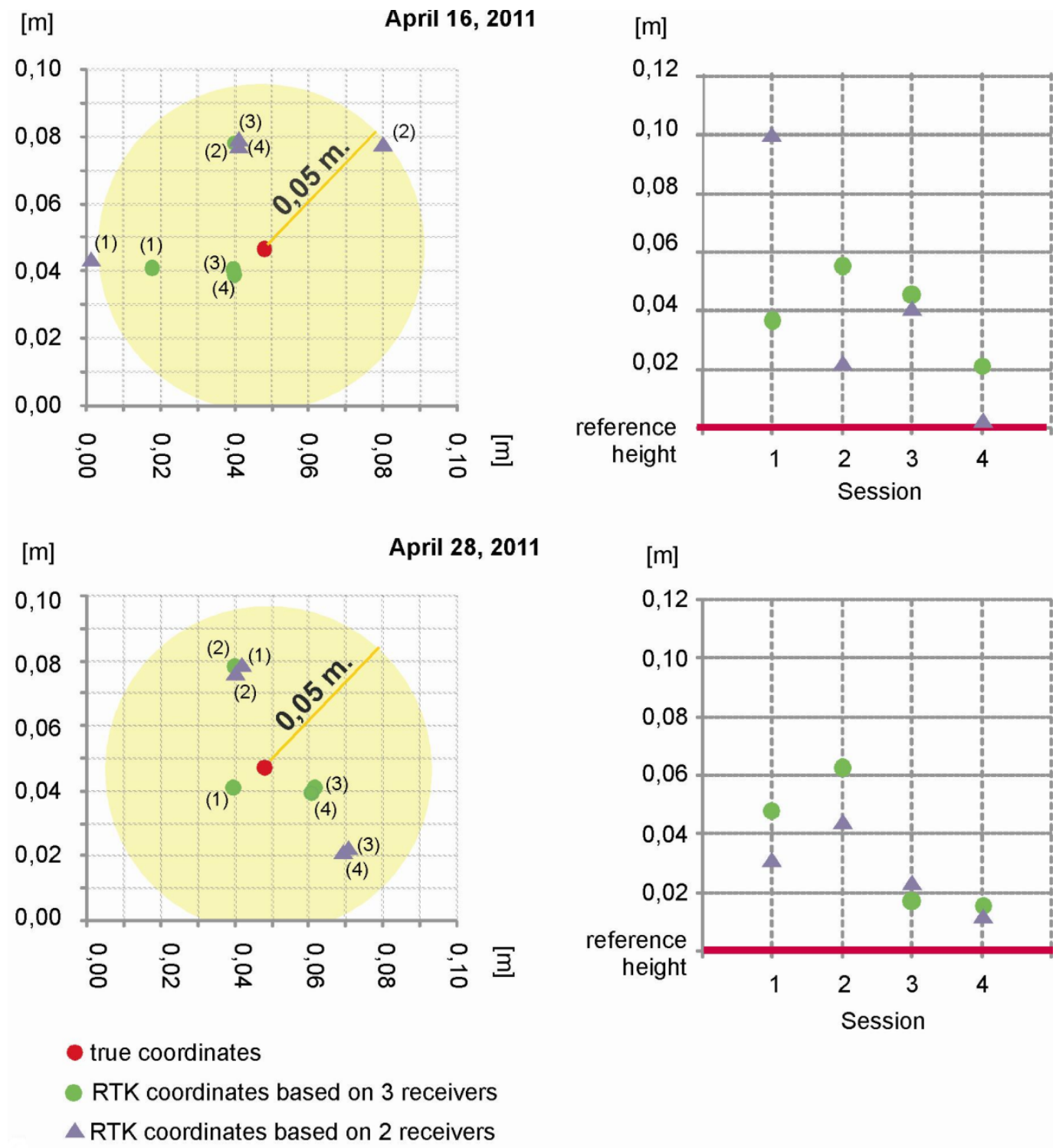

Fig. 9. Distribution of positions from the RTK-Net application using two or three GPS/RTK receivers

Although in this paper only RTK positions were investigated this approach, based on three GNSS receivers, is extremely useful for reliable and redundant rapid static positioning using the baselines determined between the NORTH, MIDDLE and SOUTH receiver as well as any VRS station located near the observed site in rapid static solutions (Bakuła, 2012). 


\section{SUMMARY}

Reliable determination of coordinates is the key issue in real time positioning. On the base of diversified experiments it can be concluded that the results of RTK surveys conducted under forest conditions is characterized by high precision from the moment of solving the ambiguity until the moment of losing that solution but considering the appearance of gross errors difficult to detect the results of such surveys are not reliable. Obtaining accurate and, first of all, reliable coordinates of forest points in RTK surveys is possible on condition of including the additional, independent control, e.g. the repeated survey. The RTK-Net positioning technique presented in this paper introduces the independent control for determination of coordinates and, additionally, allows obtaining accurate and reliable coordinates within the shortest time possible. The possibility of conducting survey using any GPS/GLONASS receivers, also by different manufacturers, is another advantage of the presented technique. The developed RTK-Net approach is highly productive in determining coordinates of control points under difficult observation conditions and it allows, first of all, obtaining reliable coordinates with the accuracy expressed in centimeters.

Acknowledgments. This work was supported by the grant funded by Polish Ministry of Science and Higher Education (No N N526 197438).

\section{REFERENCES}

Bakuła M. (2012) An approach to reliable rapid static GNSS surveying. Survey Review (in print).

Bakuła M., Kaźmierczak R., Grunwald G. (2011) Analysis of possibilities for applying the ASG-EUPOS system services for establishing the detailed control networks. Technical Sciences 14 (2), 217-228.

Bakuła M. (2010) Network code DGPS positioning and reliable estimation of position accuracy, Survey Review, 42, 315, pp. 82-91.

Bakuła M., Oszczak S., Pelc-Mieczkowska R. (2009) Performance of RTK positioning in forest conditions: case study. Journal of Surveying Engineering, American Society of Civil Engineers, Vol. 135, No 3, 125-130.

Bosy J., Graszka W., Leończyk M. (2007) ASG-EUPOS - a multifunctional precise satellite positioning system in Poland. Eur J Nav 5(4):30-34.

Dai L., Han S., Wang J., Rizos C. (2001) A study on GPS/GLONASS multiple reference station techniques for precise real-time carrier phase-based positioning. ION GPS 2001, 392-403.

Firuzabardi D., King R.W. (2011) GPS precision as a function of session duration and reference freme using Multi-point software. GPS Solution. DOI 10.1007/s10291-0110218-8.

Giménez E., Selmira Garrido M., Clara de Lacy M., Gil A.J. (2011) Comparing RTK positioning from updated REGAM and MERISTEMUM CORS networks in Southeast Spain, Journal of Applied Geodesy 5, 23-35.

Hasegawa H., Yoshimura T. (2003) Application of dual-frequency GPS receiver for static surveying under tree canopy. Journal of Forest Research, Springer-Verlag, Tokyo Inc, 8(2), 103-110.

Hu G.R., Khoo H.S., Goh P.C., Law C.L. (2003) Development and assessment of GPS virtual reference stations for RTK positioning. Journal of Geodesy, 77(5-6): 292-302. 
Lee I., Ge L. (2006) The performance of RTK-GPS for surveying under challenging environment al conditions. Earth Planets Space, 58, 515-522.

Pirti A., Gümüș K., Erkaya H., Gürsel Hoşbaş R. (2010) Evaluating repeatability of RTK GPS/GLONASS near/under forest environment. Croatian Journal of Forest Engineering, Vol 31, Issue 1, 23-33.

Teunissen P.J.G., Verhagen S. (2008) GNSS ambiguity resolution: when and how to fix or not to fix? In: Xu P, Liu J, Dermanis A (eds) VI Hotine-Marussi symposium on theoretical and computational geodesy. Springer, Berlin, 143-148.

Verhagen S. (2004) Integer ambiguity validation: an open problem? GPS Solution 8(1):3643.

Vollath U., Landau H., Chen X., Doucet K., Pagels C. (2002) Network RTK versus single base RTK -understanding the error characteristics, Proceedings of ION GPS 2002, Portland, OR, 2774-2781.

Wang Ch., Feng Y., Higgins M., Cowie B. (2010) Assessment of commercial network RTK user positioning performance over long inter-station distance. Journal of Global Positioning systems, Vol. 9. No. 1, 78-89.

Received: 2012-01-02,

Reviewed: 2012-04-02, by J. Rogowski,

Accepted: 2012-04-03. 